EPJ Web of Conferences 78, 07003 (2014)

DOI: $10.1051 /$ epjconf / 20147807003

(C) Owned by the authors, published by EDP Sciences, 2014

\title{
Colored condensates deep inside neutron stars
}

\author{
David Blaschke ${ }^{1,2}$ \\ ${ }^{1}$ University of Wroclaw (Poland) \\ ${ }^{2}$ JINR Dubna (Russia)
}

\begin{abstract}
It is demonstrated how in the absence of solutions for QCD under conditions deep inside compact stars an equation of state can be obtained within a model that is built on the basic symmetries of the QCD Lagrangian, in particular chiral symmetry and color symmetry. While in the vacuum the chiral symmetry is spontaneously broken, it gets restored at high densities. Color symmetry, however, gets broken simultaneously by the formation of colorful diquark condensates. It is shown that a strong diquark condensate in cold dense quark matter is essential for supporting the possibility that such states could exist in the recently observed pulsars with masses of $2 M_{\odot}$.
\end{abstract}

\section{Color superconductivity in the QCD phase diagram}

Since Eugene Wigner was awarded the Nobel prize for his discovery and application of fundamental symmetry principles, particularly in nuclear and elementary particle physics, our understanding of strong interactions within quantum chromodynamics (QCD) is based more than ever on these concepts. I will elucidate this statement on the example of the QCD phase diagram and the question for quark matter in compact stars. Progress has been made for understanding the mass spectrum of hadrons and also their interactions on the basis of ab-inito simulations using the QCD Lagrangian on the lattice. Extensions of such calculations to finite temperatures have provided detailed numerical data on the QCD phase transition but are limited to the domain of vanishing or low baryon densities, not applicable for the whole QCD phase diagram and in particular not for compact star interiors, see [1] for a recent overview. In this situation, effective models for low-energy QCD are investigated instead which share basic symmetries with the QCD Lagrangian and allow to study the patterns of their dynamical breaking and restoration under extreme conditions of temperature and baryon density (or chemical potential), spanning the basic axes of the QCD phase diagram, see Fig. 1. The workhorse of this kind of studies is the Nambu-Jona-Lasinio (NJL) model [2-4], recently extended by the coupling to a background gluon field in the Polyakov gauge with a meanfield Potential $\mathcal{U}$ [5],

$$
\mathcal{L}=\bar{q}\left(-i \gamma_{\mu} D_{\mu}+\hat{m}+\gamma_{0} \hat{\mu}\right) q+\mathcal{L}_{\text {int }}+\mathcal{U}(T, \Phi),
$$

representing the (approximate) chiral symmetry in the free Dirac fermion Lagrangian and the 4fermion interaction model in the scalar-pseudoscalar sector

$$
\mathcal{L}_{\text {int }}=G_{S}\left\{\sum_{a=0}^{8}\left[\left(\bar{q} \tau_{a} q\right)^{2}+\left(\bar{q} \tau_{a} i \gamma_{5} q\right)^{2}\right]+\eta_{V}\left(\bar{q} i \gamma_{0} q\right)^{2}+\eta_{D} \sum_{a=2,5,7}\left(\bar{q} i \gamma_{5} \tau_{a} \lambda_{a} C \bar{q}^{T}\right)\left(q^{T} C i \gamma_{5} \tau_{a} \lambda_{a} q\right)\right\}
$$

This is an Open Access article distributed under the terms of the Creative Commons Attribution License 4.0, which permits unrestricted use, distribution, and reproduction in any medium, provided the original work is properly cited. 
augmented here with 4-fermion couplings in the isoscalar vector and scalar diquark interaction channels with the dimensionless coupling strengths $\eta_{V}$ and $\eta_{D}$, resp., which are free parameters of the model, see [6] for details. While $\eta_{D}$ to determines the onset of the quark matter phase, $\eta_{V}$ is essential for its stiffness. The thermodynamic potential of this model in the meanfield approximation can be given the closed form

$$
\begin{aligned}
\Omega(T,\{\mu\}) & =\frac{\phi_{u}^{2}+\phi_{d}^{2}+\phi_{s}^{2}}{8 G_{S}}-\frac{\omega_{u}^{2}+\omega_{d}^{2}+\omega_{s}^{2}}{8 G_{V}}+\frac{\Delta_{u d}^{2}+\Delta_{u s}^{2}+\Delta_{d s}^{2}}{4 G_{D}} \\
& -\int \frac{d^{3} p}{(2 \pi)^{3}} \sum_{n=1}^{18}\left[E_{n}+2 T \ln \left(1+e^{-E_{n} / T}\right)\right]+\Omega_{l e p}-\Omega_{0}+\mathcal{U}(\Phi, T),
\end{aligned}
$$

where for compact star applications the lepton contribution has been added and a vacuum contribution $\left(\Omega_{0}\right)$ has been subtracted [7]. The meanfields $\phi_{f}, \Delta_{i k}$ and $\Phi=\left(1 / N_{c}\right) \operatorname{Tr}_{c} \exp \left[i \beta\left(\phi_{3} \lambda_{3}+\phi_{8} \lambda_{8}\right)\right]$ are the order parameters signalling chiral symmetry breaking $(\chi \mathrm{SB})$, color superconductivity (2SC and CFL) and deconfinement, respectively. Their values are determined by the necessary condition for a minimum of the thermodynamic potential (gap equations) under the constraint of vanishing color charge denity (the last two equations)

$$
\frac{\partial \Omega}{\partial \phi_{f}}=\frac{\partial \Omega}{\partial \Delta_{i k}}=\frac{\partial \Omega}{\partial \phi_{3}}=\frac{\partial \Omega}{\partial \phi_{8}}=0 ; \frac{\partial \Omega}{\partial \mu_{3}}=\frac{\partial \Omega}{\partial \mu_{8}}=0,
$$

that need to be solved self-consistently. The results are shown in the phase diagram of Fig. 1 (left panel), where in the plane of temperature $T$ and quark chemical potential $\mu$ the basic QCD phases are shown as regions with nonvanishing order parameters. An interesting artifact of the meanfield approximation is the occurrence of a region with negative baryon density $n_{B}<0$ which signals a thermodynamic instability [8]. It is cured by the occurrence of hadronic matter (beyond meanfield) in the region where chiral symmetry is broken and confinement prevails, see the right panel of Fig. 1 where a quark-hadron phase transition is obtained by a Maxwell construction between the meanfield quark matter model and a density-dependent relativistic meanfield model of nuclear matter. It is interesting to note that the Polyakov-loop suppression of colored quark excitations in the region of the phase diagram where $\Phi \ll 1$ leads to a strong reduction of the quark Pauli blocking effect and thus to a strong increase of the critical temperature of the color superconducting $2 \mathrm{SC}$ phase. This raises hopes that this exotic phase of QCD could be accessible [9] in future dedicated heavy-ion collision experiments like at FAIR-CBM in Darmstadt and NICA-MPD or BM@N in Dubna [10].

\section{Color superconducting phases in compact stars}

For the application of the above described low-energy QCD model to study the composition of compact star interiors one has to embody the constraints of electric neutrality and $\beta$-equilibrium to the thermodynamic potential and then use the resulting $T=0$ equation of state (EoS) for the pressure $p(\mu)=\Omega(T=0,\{\mu\})$ from which all other thermodynamic functions can be derived which are required to solve the Tolman-Oppenheimer-Volkoff equations of compact star stability. To this end, we use a hybrid EoS obtained by a Maxwell construction with the DBHF EoS for hadronic matter.

Results are shown in Fig. 2 where in the left panel sequences of compact star configurations are given in the mass $(M)$ vs. radius $(R)$ and mass vs. central baryon density $n_{B}(0)$ plane, resp., for given choices of the parameter pairs $\left(\eta_{V}, \eta_{D}\right)$ which allow to describe massive stars of the kind of the two recently discovered $2 M_{\odot}$ pulsars $[11,12]$ as hybrid stars with a color superconducting quark matter core. Note that an increase of the diquark coupling entails a lowering of the critical 

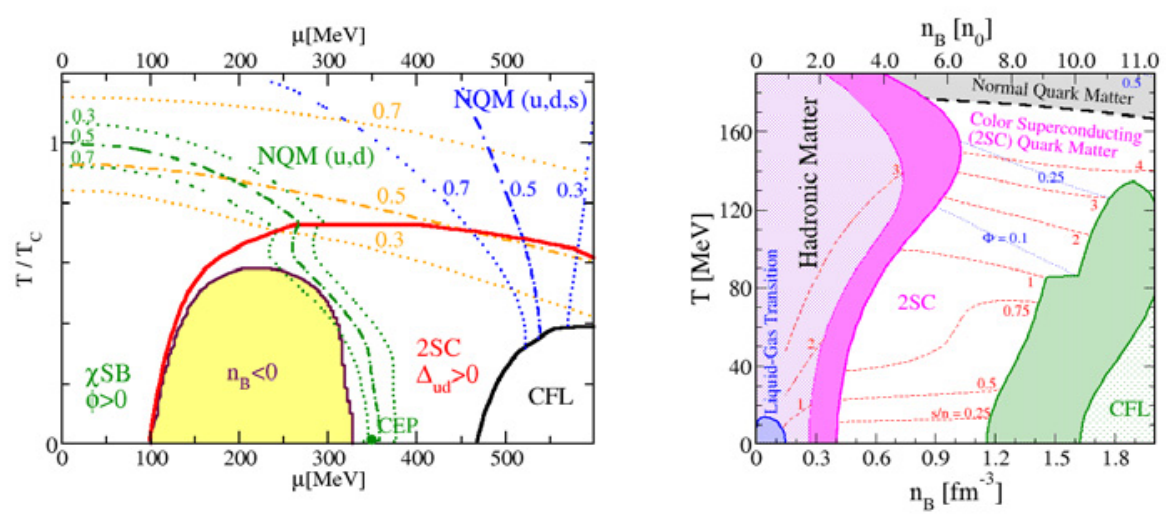

Figure 1. Left: Phase diagram of color superconducting 3-flavor PNJL model at meanfield level in the $T$ - $\mu$ plane with a thermodynamic instability $n_{B}<0$ in the coexistence region of 2SC and $\chi \mathrm{SB}$ phases. Right: Phase diagram with first order transition (Maxwell construction) to hadronic matter described in the DD2 model.
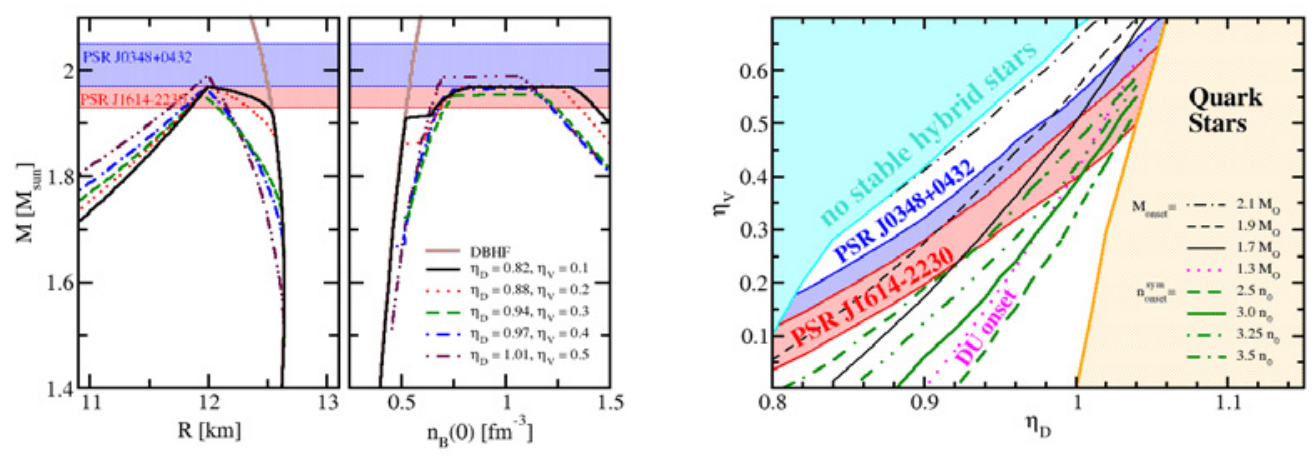

Figure 2. Left: Mass-radius and mass-central density sequences for parameter sets allowing hybrid stars which fulfill the $2 M_{\odot}$ mass constraints. Right: Region of parameter values for the vector meson $\left(\eta_{V}\right)$ and scalar diquark $\left(\eta_{D}\right)$ coupling strengths where stable hybrid stars with quark matter cores are possible (between cyan and orange domains) and where the $2 M_{\odot}$ mass constraints for the pulsars PSR J0348+0432 (blue) and PSR J1614-2230 (red) are fulfilled.

density for the onset of 2SC quark matter but requires a simultaneous stiffening by an increase of the vector coupling in order to still allow a sufficiently high maximum mass in accordance with the measurements $[11,12]$. A second observation is that the maximum allowable mass of a sequence is determined by the onset of strange quark matter formation (CFL phase) which softens the EoS so much that all configurations with strange quark matter cores lie on an unstable branch. The results of these systematic studies within the (P)NJL model for quark matter [13] are summarized in the right panel of Fig. 2. A conclusion drawn from this study is that should the high-mass pulsars be hybrid stars with a quark matter core the latter has to be in color superconducting phase! Another 
conclusion from such a study is that the critical density for the deconfinement transition in heavy-ion collisions (symmetric nuclear matter) should exceed 3.5 saturation densities unless for the diquark coupling holds $\eta_{V}>1.0$. The appearance of quark matter in the cores of compact stars is a possibility to solve problems that occur with purely hadronic scenarios such as the direct Urca cooling problem [14] and the hyperon puzzle [15]. The question arises: Can one detect by observations of compact stars a deconfinement phase transition in their interior? Recently, it has been suggested that: yes, if our two high-mass pulsars would be so-called "mass twins", i.e. have very different radii so that the more compact twin would be the one with a quark matter interior while the larger star would consist of hadronic matter - this would be possible at the same high mass only with a strong phase transition with sufficiently large latent heat [16]! Such transitions with a coexistence of two phases are known to produce structures like bubbles and droplets (pasta phase) of different shapes and sizes governed by surface tension and Coulomb effects, see [17] and references therein. For the calculation of such pasta phases one uses the very successful concept of the Wigner-Seitz cell [18]. A strong QCD phase transition might contribute to solving one of the long-standing puzzles in Astrophysics: the quest for a supernova explosion mechanism [19].

\section{Acknowledgements}

Inspiring discussions with many colleagues and the high intellectual spirit of this Conference celebrating Eugene Wigners $111^{\text {th }}$ birthday are gratefully acknowledged. This work was supported by NCN under grant no. UMO-2011/02/A/ST2/00306 and by MNiSW under grant no. 1009/S/IFT/14.

\section{References}

[1] F. Karsch, Acta Phys. Polon. Supp. 7, no. 1, 117 (2014).

[2] S. P. Klevansky, Rev. Mod. Phys. 64, 649 (1992).

[3] T. Hatsuda and T. Kunihiro, Phys. Rept. 247, 221 (1994).

[4] M. Buballa, Phys. Rept. 407, 205 (2005).

[5] K. Fukushima and C. Sasaki, Prog. Part. Nucl. Phys. 72, 99 (2013).

[6] G. A. Contrera, A. G. Grunfeld and D. B. Blaschke, Phys. Part. Nucl. Lett. 11, 342 (2014).

[7] D. Blaschke, S. Fredriksson, H. Grigorian, A. M. Öztas and F. Sandin, Phys. Rev. D 72, 065020 (2005).

[8] D. Gomez Dumm, D. B. Blaschke, A. G. Grunfeld and N. N. Scoccola, Phys. Rev. D 78, 114021 (2008).

[9] D. B. Blaschke, F. Sandin, V. V. Skokov and S. Typel, Acta Phys. Polon. Supp. 3, 741 (2010).

[10] T. Klähn, D. Blaschke and F. Weber, Phys. Part. Nucl. Lett. 9, 484 (2012).

[11] P. Demorest et al., Nature 467, 1081 (2010).

[12] J. Antoniadis et al., Science 340, 6131 (2013).

[13] T. Klähn, D. B. Blaschke and R. Łastowiecki, Phys. Rev. D 88, 085001 (2013).

[14] T. Klähn et al., Phys. Lett. B 654, 170 (2007).

[15] R. Lastowiecki, D. Blaschke, H. Grigorian and S. Typel, Acta Phys. Polon. Supp. 5, 535 (2012).

[16] D. Blaschke, D. E. Alvarez-Castillo and S. Benic, PoS CPOD 2013, 063 (2013).

[17] N. Yasutake et al., Phys. Rev. C 89, 065803 (2014).

[18] E. Wigner and F. Seitz, Phys. Rev. 43, 804 (1933).

[19] T. Fischer, T. Klähn, I. Sagert, M. Hempel and D. Blaschke, Acta Phys. Polon. Supp. 7, 153 (2014). 